\title{
NON-LINEAR VIBRATIONS OF THREE-LAYER BEAMS WITH VISCOELASTIC CORES I. THEORY ${ }^{\dagger}$
}

\author{
M. W. HYER \\ Department of Mechanical Engineering and Mechanics, \\ Old Dominion University, Norfolk, Virginia 23508, U.S.A. \\ W. J. ANDERSON \\ Department of Aerospace Engineering, University of Michigan \\ AND \\ R. A. SCOTT \\ Department of Applied Mechanics and Engineering Science, \\ University of Michigan, Ann Arbor, Michigan 48104, U.S.A.
}

(Received 9 August 1975, and in revised form 29 November 1975)

\begin{abstract}
Approximate equations of motion are developed for large amplitude motions of threelayer axially restrained unsymmetrical beams with viscoelastic cores. The external force consists of a constant plus an oscillatory term. The combination of this form of forcing and the large amplitude motions cause the beam to respond at multiples of the forcing frequency. This can lead to difficultics in the complex modulus approach to viscoelasticity. These are overcome here through use of hereditary integrals and their relationships with complex moduli. Theoretical results on the frequency response of clamped, symmetrical beams are compared with earlier experimental work. On the whole, reasonable agreement is found.
\end{abstract}

\section{INTRODUCTION}

Considerable theoretical and experimental work on the linear vibration of sandwich structures with elastic components has been done over the years (see the paper by Habip [1] for a thorough survey of progress made prior to 1965). A major contributor was $\mathrm{Yu}[2-4]$. He developed a general one-dimensional theory for plates, a theory which incorporated the effects of transverse shear deformations and rotatory inertia in both core and face sheets. He also presented a simplified version for two-dimensional motions of sandwich plates with identical face sheets and soft cores. Krajcinovic [5, 6] also presented results on symmetric, three-layer elastic beams using an approach similar to Yu's. However, his approach was unique in that he chose orthogonal displacement functions for the beam as a whole, as a result of which several governing equations were uncoupled. Recently Folie [7] did further work in this area. He developed a theory for the transverse bending of three-layer plates with isotropic outer layers and an orthotropic core. Chan and Cheung [8] numerically solved problems of bending and vibration of multi-layered plates by what is termed the finite strip method. Ahmed [9] used a finite-element method to obtain information on the free vibrations of curved, sandwich beams. Recently, Krishna Murty and Shimpi [10] developed a theory for laminated beams which includes the effects of bending and shear, rotatory and longitudinal inertia, in

† Work funded in part by the National Science Foundation (Grant No. GK-36374X). It forms part of a Ph.D. thesis of the first author in Aerospace Engineering at the University of Michigan, 1974. 
all laminates. Of course, there is now a large body of literature concerning theories for elastic sandwich structures with a large number of components (see the paper by Ben-Amoz [11] for more detail).

Considerable interest has developed in using viscoelastic materials in sandwich configurations, both constrained and unconstrained, as means of reducing vibration levels (see, e.g., the paper by Plunkett [12]). DiTaranto [13] developed linear equations for vibrations of layered beams of finite length, loss factors and natural frequencies being computed by DiTaranto and Blasingame [14]. Jones, Salerno and Savacchio [15] did an analytical and experimental evaluation of damping for such beams, the agreement between theory and experiment being at best fair. Yin, Kelly and Barry [16] presented an experimental study of the effects of constrained layer damping on plates, beams, tubes, and other structural members. Mead and Markus [17, 18] and Mead and DiTaranto [19] gave results on loss factors and resonant frequencies for three-layer sandwich beams. Lu and Douglas [20], in experiments on mechanical impedance, got excellent agreement with results obtained by using the analytical formulation of Mead and Markus [17].

Most of these investigations revealed that core materials made from commonly available viscoelastic materials damped vibration only over a limited frequency range. Grootenhuis [21], Agbasiere and Grootenhuis [22], and Nakra and Grootenhuis [23] looked at sandwiches involving several viscoelastic layers, each one having its peak damping in a different frequency range. Grootenhuis [24] showed that certain unsymmetrical sandwich structures provided more effective damping over a wider frequency range as compared to their symmetric counterparts.

A common feature of the theories involved in the above works was to assume a displacement field for each layer. Governing equations were then arrived at by looking at the equilibrium of a beam element, together with the layer materials constitutive laws and the interface conditions. Recently, Yan and Dowell $[25,26]$ used the principle of virtual work to arrive at general linear equations for the dynamics of three-layer sandwich plates. Restricting attention to soft cores and ignoring all inertias except the transverse one, they derived dispersion relations which agreed quite well with the full equations as well as with the results given by Mead, Markus and DiTaranto. Yan and Dowell also found good agreement between experiments they performed and their theory for beams.

Work related to the above, that the present authors are aware of, should be cited for completeness: Asnani and Nakra [27], Braunisch [28], Chandrasekharan and Ghosh [29], DiTaranto and McGraw [30], Emerson [31], Jones and Parin [32], O. Markus and S. Markus [33], Nakra [34], Sadasiva Rao [35], and Torvik and Strickland [36].

Even though considerable work has been done on the non-linear dynamics of continuous media (for example, that of Ho, Scott and Eisley [37]), relatively little has been in the area of sandwich structures. Reissner [38], in a study on the static deflection of plates with membrane face sheets, showed that, for core materials with elastic moduli the same order of magnitude as those for the face material, linear theory is adequate provided that the transverse deflections are small compared to the total plate thickness. However, as the core becomes softer and softer, the range of linear behavior decreases and geometric non-linearities become important. The same behavior is anticipated for the dynamic response of sandwich structures with membrane face layers as well as for structures with face layers too thick to be considered membranes. Yu $[39,40]$ extended his linear analysis of flexural vibrations of elastic sandwich plates with thin face sheets to include geometric non-linearities. Wempner and Baylor [41] also derived equations for the large amplitude motions of elastic sandwich plates with weak cores. Habip [42] used perturbation methods to derive equations for the static deflection of two-layer plates. The first-order terms are Von Karman equations, whereas the second-order terms reflect shear effects. Bert [43] has also contributed to the area. 


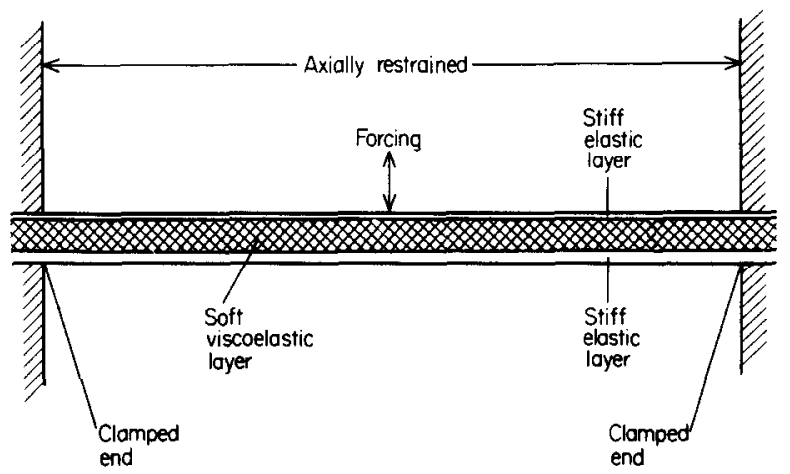

Figure 1. Beam configuration.

In one of the few studies which included experimental results, and the major motivation for the present study, Kovac, Anderson and Scott [44] investigated large amplitude vibrations of harmonically forced symmetric sandwich beams with viscoelastic cores and identical thin face sheets. The ends of the beam were restrained from moving towards cach other, and so the transverse vibration induced axial stretching. The face sheets were considered as elastic membranes while the constitutive properties of the core were represented by complex moduli evaluated at the forcing frequency.

In general, agreement between theory and experiment was reasonable. However, the experiment seemed to reveal a superharmonic response when the beam was forced in the vicinity of one-half its linear natural frequency. In this region, the experimental frequency response, as well as the mode shape, deviated considerably from the theoretical predictions. Figure 1 shows the beam configuration and Figure 2 shows results of the investigation.

This paper is the first of two papers summarizing the work directed at explaining the deviation. The prime concern was to determine whether the superharmonic was a structural effect or whether it was due to something in the experiment not accounted for in the theory. Experimentally [44], the beam was forced with an electromagnetic device. When alternating current is passed through an electromagnet, the force felt by a ferromagnetic object is actually a constant bias force plus an alternating force. This bias force effect was not accounted for in the theory. Bennett and Eisley [45], in similar work on homogeneous beams, showed the effect to be negligible, whilc Meirovitch [46] showed considerable superharmonic could exist

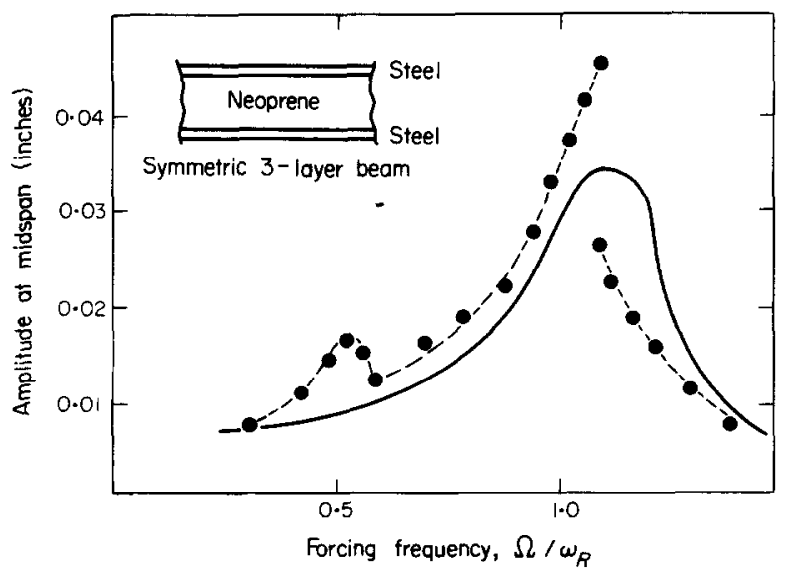

Figure 2. Comparison between theory and experiment from reference [44]. _ _, Theory; -- --, experiment; $\omega_{R}=$ linear resonant frequency. 
when forcing a cubic hardening system with a constant plus a harmonic force. Of equal importance was the fact that since the superharmonic response did exist, using complex moduli evaluated at the forcing frequency to represent the core material properties was not proper. Thus, in a first attempt to explain the deviation, the response of a three-layer sandwich beam, with a viscoelastic core, to a constant bias force plus a harmonic force was obtained. A summary of that work is presented here. The inclusion of a bias force did not fully account for the superharmonic behavior reported in reference [44] and so it was necessary to conduct a thorough examination of the experimental procedures used in that work and similar investigations. It was found that deficiencies in the experimental study of superharmonic response needed to be corrected. That examination will be presented in a companion paper.

In the present paper the equations governing the non-linear vibrations of a three-layer beam with a viscoelastic core are derived. The equations are valid for beams with dissimilar face layers and are not restricted to beams with membrane face layers. The constitutive properties of the viscoelastic core are treated by using relations between the hereditary integrals and the complex moduli. An approximate solution scheme is presented and numerical results are obtained and compared with the experimental results of reference [44].

\section{DEVELOPMENT OF THE EQUATIONS OF MOTION}

The equations of motion are derived by using the principle of virtual work. By using a summation convention, the principle of virtual work can be stated as

$$
\begin{aligned}
& \int_{V}\left[\rho F_{j}+\frac{\partial}{\partial x_{k}}\left[S_{k m}\left(\delta_{j m}+e_{j m}+\omega_{j m}\right)\right]\right] \delta u_{j} \mathrm{~d} V+ \\
& \quad+\int_{S}\left[T_{j}-S_{k m}\left(\delta_{j m}+e_{j m}+\omega_{j m}\right) v_{k}\right] \delta u_{j} \mathrm{~d} S=0,
\end{aligned}
$$

where $V$ and $S$ denote the volume and surface of the material in the undeformed state, $\rho$ is the density, $F_{j}$ the body force per unit mass, $S_{k m}$ are Kirchhoff stress components (forces per unit undeformed area), $T_{j}$ are surface tractions (per unit undeformed area), $\delta_{j m}$ stands for the Kronecker delta, $\delta u_{j}$ denotes virtual displacements, $v_{k}$ are the direction cosines of the unit outward normal to $S$, and

$$
\begin{aligned}
& e_{j m}=\frac{1}{2}\left(\partial u_{j} / \partial x_{m}+\partial u_{m} / \partial x_{j}\right), \\
& \omega_{j m}=\frac{1}{2}\left(\partial u_{j} / \partial x_{m}-\partial u_{m} / \partial x_{j}\right),
\end{aligned}
$$

where $x_{j}$ are a set of Cartesian co-ordinates designating a point in the undeformed body. It should be noted at the outset that the theory under development is not aimed at very large deflections. The theory is geared towards deflections of the order of the beam thickness and consequently there will be no distinction between volumes and surfaces before and after deformation. Non-linearity enters because of geometric constraints and will make its presence felt by using Green's non-linear strain measures [47]:

$$
\varepsilon_{r s}=e_{r s}+\frac{1}{2}\left(e_{k r}+\omega_{k r}\right)\left(e_{k s}+\omega_{k s}\right) .
$$

Based on experience with homogeneous beam and plate theory, and experience of others, such as $\mathrm{Yu}$ and $\mathrm{Yan}$ and Dowell, in sandwich constructions, it was felt that a viable theory would be obtained based on the following assumptions: (i) referring to Figure 3, and switching permanently to $x y z$-notation, one takes all physical quantities to be independent of $y$; (ii) the normal strains in the thickness direction are negligible; (iii) the normal stresses in the thickness directions are small compared to other stresses; (iv) in equation (4), $e_{i j}$ can be 


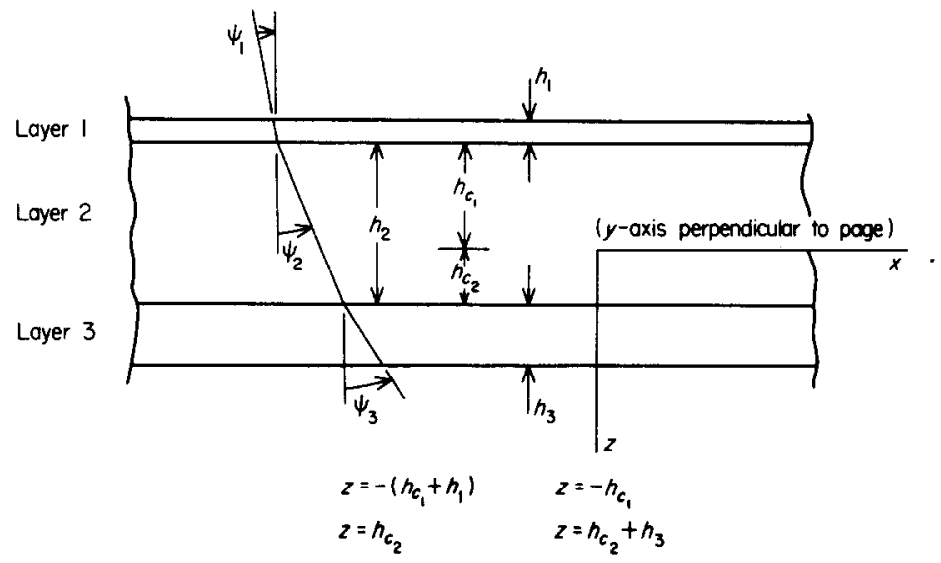

Figure 3. Beam geometry-origin of co-ordinates at mid-span.

dropped compared to $\omega_{i j}$ and both can be neglected compared to unity; (v) in each layer, plane sections are taken to remain plane. In a layer, the in-plane displacements are due to extension, shear and bending. The extensional displacement is assumed uniform across the thickness, while the shear and bending are assumed to give rise to a linear variation. In keeping with the assumption of zero normal strain, the transverse displacement is assumed to be the same for each layer. In summary:

$$
\begin{gathered}
u_{i}(x, z, t)=u_{i}^{0}(x, t)+z \psi_{i}(x, t), \quad i=1,2,3, \\
w_{i}(x, z, t)=w(x, t), \quad i=1,2,3,
\end{gathered}
$$

where $u_{i}^{0}$ is the extensional in-plane displacement, $\psi_{i}$ the shear and bending angle (see Figure 3 ), $w$ the transverse displacement and the index $i$ refers to the layer in question (it will be reserved henceforth for that purpose throughout this work).

Upon using the above-listed assumptions and equations (5) and (6), the principle of virtual work gives, on integrating w.r.t. $z$,

$$
\begin{aligned}
& \sum_{i=1}^{3} \int_{-L / 2}^{L / 2}\left\{\left[-\rho_{i}\left(h_{i} \ddot{u}_{i}^{0}+A_{i 0} \ddot{\psi}_{i}\right)+\frac{\partial}{\partial x}\left(N_{x x i}+Q_{x z i} \omega_{x z i}\right)+\left.\left(S_{z x i}+S_{z z i} \omega_{x z i}\right)\right|_{z_{i-1}} ^{z_{i}}\right] \delta u_{i}^{0}+\right. \\
& \quad+\left[-\rho_{i}\left(A_{i 0} \ddot{u}_{i}^{0}+I_{i 0} \ddot{\psi}_{i}\right)+\frac{\partial}{\partial x}\left(M_{x x i}+M_{x z i} \omega_{x z i}\right)-Q_{x z i}+\left.\left(z\left(S_{z x i}+S_{z z i} \omega_{x z i}\right)\right)\right|_{z i-1} ^{z_{i}}\right] \delta \psi_{i}+ \\
& \left.\quad+\left[-\rho_{i} h_{i} \ddot{w}_{i}+\frac{\partial}{\partial x}\left(N_{x x i} \omega_{z x i}+Q_{x z i}\right)+\left.\left(S_{z x i} \omega_{z x i}+S_{z z i}\right)\right|_{z_{i-1}} ^{z_{i}}\right] \delta w\right) \mathrm{d} x+\text { boundary term }=0
\end{aligned}
$$

where the moment coefficients, $h_{i}, A_{i 0}$ and $I_{i 0}$, and the stress resultants are given by

$$
\begin{aligned}
& h_{i}=\int_{z_{i-1}}^{z_{i}} \mathrm{~d} z, \quad A_{i 0}=\int_{z_{i-1}}^{z_{1}} z \mathrm{~d} z, \quad I_{i 0}=\int_{z_{i-1}}^{z_{i}} z^{2} \mathrm{~d} z, \\
& N_{x x i}=\int_{z_{i-1}}^{z_{i}} S_{x x l} \mathrm{~d} z, \quad M_{x x i}=\int_{z_{i-1}}^{z_{i}} z S_{x x i} \mathrm{~d} z, \quad Q_{x z i}=\int_{z_{i-1}}^{z_{i}} S_{x z l} \mathrm{~d} z, \\
& M_{x z l}=\int_{z_{i-1}}^{z_{i}} z S_{x z l} \mathrm{~d} z .
\end{aligned}
$$


In the interests of brevity the boundary term in equation (7) has not been written out explicitly since in the ultimate application it will vanish. The term is written out in detail in reference [48].

Upon noting that, at this point, each layer in the sandwich structure is being treated separately and thus $\delta u_{l}^{0}, \delta \psi_{i}$ and $\delta w$ are independent, equation (7) yields

$$
\begin{gathered}
\sum_{i=1}^{3}\left\{\frac{\partial}{\partial x}\left(N_{x x i}+Q_{x z i} \omega_{x z i}\right)+\left.\left(S_{z x i}+S_{z z i} \omega_{x z i}\right)\right|_{z_{i-1}} ^{z_{i}}-\rho_{i}\left(h_{i} \ddot{u}_{i}^{0}+A_{i 0} \ddot{\psi}_{i}\right)\right\}=0 \\
\sum_{i=1}^{3}\left\{\frac{\partial}{\partial x}\left(M_{x x i}+M_{x z i} \omega_{x z i}\right)+\left.\left(z\left(S_{z x i}+S_{z z i} \omega_{x z i}\right)\right)\right|_{z_{i-1}} ^{z_{i}}-Q_{x z i} \cdots \rho_{i}\left(A_{i 0} \ddot{u}_{i}^{0}+I_{i 0} \ddot{\psi}_{i}\right)\right\}=0 \\
\sum_{i=1}^{3}\left\{\frac{\partial}{\partial x}\left(N_{x x i} \omega_{z x i}+Q_{x z i}\right)+\left.\left(S_{z x i} \omega_{z x i}+S_{z z i}\right)\right|_{z_{i-1}} ^{z_{i}}-\rho_{i} h_{i} \ddot{w}\right\}=0 .
\end{gathered}
$$

However, due to interface conditions, cquations (9), (10) and (11) are not all independent. Upon specializing to perfect bonds, displacement continuity requires

$$
\begin{aligned}
& u_{1}\left(x, z_{1}, t\right)=u_{2}\left(x, z_{1}, t\right), \\
& u_{2}\left(x, z_{2}, t\right)=u_{3}\left(x, z_{2}, t\right) .
\end{aligned}
$$

As $S_{z z i}$ has been taken to be small, equations (12) and (13) require continuity of the shear stress at the interfaces (as can be shown from the boundary term):

$$
\begin{aligned}
& S_{x z 1}\left(x, z_{1}, t\right)=S_{z x 2}\left(x, z_{1}, t\right), \\
& S_{z x 2}\left(s, z_{2}, t\right)=S_{z x 3}\left(x, z_{2}, t\right) .
\end{aligned}
$$

Upon eliminating the interface terms by means of equations (12), (13), (14) and (15), equations (9), (10) and (11) yield

$$
\begin{aligned}
& \quad \frac{\partial}{\partial x}\left(N_{x x 1}+N_{x x 2}+N_{x x 3}+Q_{x z 1} \omega_{x z 1}+Q_{x z 2} \omega_{x z 2}+Q_{x z 3} \omega_{x z 3}\right)+\left(S_{z x 3}+S_{z x 3} \omega_{x z 3}\right)_{z_{0}}- \\
& \quad-\left(S_{z x 1}+S_{z z 1} \omega_{x z 1}\right)_{z_{0}}-\rho_{1}\left(h_{1} \ddot{u}_{1}^{0}+A_{10} \ddot{\psi}_{1}\right)-\rho_{2}\left(h_{2} \ddot{u}_{2}^{0}+A_{20} \ddot{\psi}_{2}\right) \\
& \quad-\rho_{3}\left(h_{3} \ddot{u}_{3}^{0}+A_{30} \ddot{\psi}_{3}\right)=0 \\
& \frac{\partial}{\partial x}\left(M_{x x 1}+M_{x x 2}+M_{x x 3}+M_{x z 1} \omega_{x z 1}+M_{x z 2} \omega_{x z 2}+M_{x z 3} \omega_{x z 3}\right)+\left[z\left(S_{z x 3}+S_{z z 3} \omega_{x z 3}\right)\right] z_{3}- \\
& -\left[z\left(S_{z x 1}+S_{z z 1} \omega_{x z 1}\right)\right] z_{0}-Q_{x z 1}-Q_{z x 2}-Q_{x z 3}-\rho_{1}\left(A_{10} \ddot{u}_{1}^{0}+I_{10} \ddot{\psi}_{1}\right) \\
& -\rho_{2}\left(A_{20} \ddot{u}_{2}^{0}+I_{20} \ddot{\psi}_{2}\right)-\rho_{3}\left(A_{30} \ddot{u}_{3}^{0}+I_{30} \ddot{\psi}_{3}\right)=0 \\
& \quad \frac{\partial}{\partial x}\left(N_{x x 1} \omega_{x z 1}+N_{x x 2} \omega_{z x 2}+N_{x x 3} \omega_{z x 3}+Q_{x z 1}+Q_{x z 2}+Q_{x z 3}\right)+\left(S_{z x 3} \omega_{z x 3}+S_{z z 3}\right) z_{3}- \\
& \quad-\left(S_{z x 1} \omega_{z x 1}+S_{z z 1}\right) z_{0}-\left(\rho_{1} h_{1}+\rho_{2} h_{2}+\rho_{3} h_{3}\right) \ddot{w}=0
\end{aligned}
$$

where the terms $\left(S_{z x 3}+S_{z z 3} \omega_{x z 3}\right) z_{3}$, etc., can be related to the applied tractions $T_{x 3}$, etc.

For the beams that are the subject of the ultimate study, some further simplifications can be made in equations (16), (17) and (18). Attention will be confined to soft cores and $S_{x x 2}$ (and consequently $M_{x x 2}, N_{x x 2}$ ) is taken to be negligible. The loadings will be such that transverse motion dominates and rotatory and in-plane inertia will be deleted. Finally, since the work focuses on axially restrained beams, the in-plane stress resultants $N_{x x 1}$, etc., are large, compared to the shear forces. Consequently, terms such as $Q_{x z 1} \partial \omega_{x z 1} / \partial x$ and $\omega_{x z 1} \partial Q_{x z 1} / \partial x$, 
termed shear curvature and shear buoyancy, by Chu and Herrmann [49], will be deleted in the sequel.

At this stage it is appropriate to consider the constitutive laws for the layer materials. With the materials in the faces taken to be linear, isotropic, elastic solids, Hooke's law gives, for the only non-vanishing stress components,

$$
\begin{aligned}
& S_{x x i}=E_{i} \varepsilon_{x x i}, \quad i=1,3, \\
& S_{x z i}=2 G_{i} \varepsilon_{x z i}, \quad i=1,3,
\end{aligned}
$$

where $E_{i}$ and $G_{l}$ stand for the Young's modulus and shear modulus, respectively. With linear viscoelastic behavior assumed, the constitutive law for the core is given by a hereditary integral

$$
S_{x z 2}=2 \int_{-\infty}^{t} Y_{2}(t-\tau) \mathrm{d} \varepsilon_{x z 2}(\tau),
$$

where $Y_{2}$ is the shear relaxation function of the material.

Some further approximations can be meaningfully made at this step. Upon using the strain measures, equations (2), (3) and (4), the assumed displacement field, equations (5) and (6), and the constitutive laws, equations (19), (20) and (21), the interface conditions represent four relations among the seven unknown displacements $u_{i}^{0}, \psi_{i}$ and $w$. The seven displacements involve kinematic terms such as

$$
\left(\psi_{2}+\partial w / \partial x\right)-\left(1 / G_{3}\right) \int_{-\infty}^{t} Y_{2}(t-\tau) \mathrm{d}\left(\psi_{2}+\partial w / \partial x\right)
$$

If the core were elastic, item (22) would be

$$
\left(\psi_{2}+\partial w / \partial x\right)-\left(G_{2} / G_{3}\right)\left(\psi_{2}+\partial w / \partial x\right),
$$

where $G_{2}$ is the shear modulus of the core. For soft cores $G_{2} / G_{3} \ll 1$ and the second term in item (23) can be deleted. By analogy, for the viscoelastic materials considered here, the following kinematic approximation to item (22) is justified:

$$
\left(\psi_{2}+\partial w / \partial x\right)-\left(1 / G_{3}\right) \int_{-\infty}^{t} Y_{2}(t-\tau) \mathrm{d}\left(\psi_{2}+\partial w / \partial x\right)=\left(\psi_{2}+\partial w / \partial x\right)
$$

Upon using this and similar approximations (more detail can be found in reference [48]) the displacement fields become

$$
\begin{gathered}
u_{1}=u+z_{1} \psi+\left(z_{1}-z\right) \partial w / \partial x \\
u_{2}=u+z \psi \\
u_{3}=u+z_{2} \psi+\left(z_{2}-z\right) \partial w / \partial x
\end{gathered}
$$

where $u \equiv u_{2}^{0}$ and $\Psi \equiv \Psi_{2}$. Also the approximations lead to

$$
\psi_{1}=-\partial w / \partial x=\psi_{3}
$$

Upon using equations (24) through (28), the strain components can be calculated from equations (2), (3) and (4). Then the stress resultants can be determined from equations (8). Substituting their values into the differential equations (16), (17) and (18), and choosing the origin of $z$ to be such that

$$
E_{3} h_{3} h_{c 2}=E_{1} h_{1} h_{c 1},
$$


one obtains

$$
\frac{\partial}{\partial x}\left\{\left(E_{3} h_{3}+E_{1} h_{1}\right)\left[\frac{\partial u}{\partial x}+\frac{1}{2}\left(\frac{\partial w}{\partial x}\right)^{2}\right]-\left[E_{3} h_{3} h_{c 2}\left(1+\frac{h_{3}}{2 h_{c 2}}\right)-E_{1} h_{1} h_{c 1}\left(1+\frac{h_{1}}{2 h_{c 1}}\right)\right] \frac{\partial^{2} w}{\partial x^{2}}\right\}=0
$$

$$
\begin{gathered}
\frac{\partial}{\partial x}\left\{\left[E_{3} h_{3} h_{c 2}\left(1+\frac{h_{3}}{2 h_{c 2}}\right)-E_{1} h_{1} h_{c 1}\left(1+\frac{h_{1}}{2 h_{c 1}}\right)\right]\left[\frac{\partial u}{\partial x}+\frac{1}{2}\left(\frac{\partial w^{2}}{\partial x}\right)\right]+\right. \\
+\left[E_{3} h_{3} h_{c 2}^{2}\left(1+\frac{h_{3}}{2 h_{c 2}}\right)+E_{1} h_{1} h_{c 1}^{2}\left(1+\frac{h_{1}}{2 h_{c 1}}\right)\right]\left(\frac{\partial \psi}{\partial x}+\frac{\partial^{2} w}{\partial x^{2}}\right)- \\
\left.-\left[E_{3} h_{3} h_{c 2}^{2}\left(1+\frac{h_{3}}{h_{c 2}}+\frac{h_{3}^{2}}{3 h_{c 2}^{2}}\right)+E_{1} h_{1} h_{c 1}^{2}\left(1+\frac{h_{1}}{h_{c 1}}+\frac{h_{1}^{2}}{3 h_{c 1}^{2}}\right)\right] \frac{\partial^{2} w}{\partial x^{2}}\right\}- \\
-h_{2}\left(1+\frac{h_{1}}{h_{2}}+\frac{h_{3}}{h_{1}}\right) \int_{-\infty}^{t} Y_{2}(t-\tau) \mathrm{d}\left(\psi+\frac{\partial w}{\partial x}\right)=0 \\
\frac{\partial}{\partial x}\left\{\left(E_{3} h_{3}+E_{1} h_{1}\right)\left[\frac{\partial u}{\partial x}+\frac{1}{2}\left(\frac{\partial w}{\partial x}\right)^{2}\right]-\left[E_{3} h_{3} h_{c 2}\left(1+\frac{h_{3}}{2 h_{c 2}}\right)-\right.\right. \\
\left.\left.-E_{1} h_{1} h_{c 1}\left(1+\frac{h_{1}}{2 h_{c 1}}\right)\right] \frac{\partial^{2} w}{\partial x^{2}} \frac{\partial w}{\partial x}\right\}+h_{2}\left(1+\frac{h_{1}}{h_{2}}+\frac{h_{3}}{h_{2}}\right) \int_{-\infty}^{t} Y_{2}(t-\tau) \mathrm{d}\left(\psi+\frac{\partial w}{\partial x}\right)+ \\
+q(x, t)=\left(\rho_{1} h_{1}+\rho_{2} h_{2}+\rho_{3} h_{3}\right) \ddot{w},
\end{gathered}
$$

where $q$ is the transverse load per unit length.

Integrating equation (30) twice with respect to $x$ and considering a fixed end beam with axial restraints, i.e.,

$$
u\left(\frac{L}{2}, t\right)=0=u\left(-\frac{L}{2}, t\right)=\frac{\partial w}{\partial x}\left(\frac{L}{2}, t\right)=\frac{\partial w}{\partial x}\left(-\frac{L}{2}, t\right),
$$

one obtains, after some manipulation

$$
\frac{\partial u}{\partial x}+\frac{1}{2}\left(\frac{\partial w}{\partial x}\right)^{2}=\left[\left(E_{3} h_{3}^{2}-E_{1} h_{1}^{2}\right) / 2\left(E_{1} h_{1}+E_{3} h_{3}\right)\right] \frac{\partial^{2} w}{\partial x^{2}}+\frac{1}{2 L} \int_{-L / 2}^{L / 2}\left(\frac{\partial w}{\partial x}\right)^{2} \mathrm{~d} x
$$

A rather interesting feature can be seen in equation (34). For unsymmetric beams it is seen that the in-plane displacement is coupled to the out-of-plane displacement through firstorder terms. However, for symmetric beams, i.e., $E_{1}=E_{3}, h_{1}=h_{3}$, then the first term on the right side of equation (34) drops and coupling is through a second-order effect.

Upon using equation (34), the differential equations (31) and (32) become

$$
\begin{gathered}
\frac{\partial}{\partial \chi}\left[K_{11} \frac{\partial^{2} \bar{w}}{\partial \chi^{2}}+K_{12}\left(\frac{\partial \bar{\psi}}{\partial \chi}+\frac{\partial^{2} \bar{w}}{\partial \chi^{2}}\right)-K_{13} \frac{\partial^{2} \bar{w}}{\partial \chi^{2}}\right]-h_{2} \int_{-\infty}^{t} Y_{2}(t-\tau) \mathrm{d}\left(\bar{\psi}+\frac{\partial \bar{w}}{\partial \chi}\right)=0 \\
K_{15}\left(\int_{-1 / 2}^{1 / 2}\left(\frac{\partial \bar{w}}{\partial \chi}\right)^{2} \mathrm{~d} \chi\right) \frac{\partial \bar{w}}{\partial \chi}+h_{2} \int_{-\infty}^{t} Y_{2}(t-\tau) \mathrm{d}\left(\frac{\partial \bar{\psi}}{\partial \chi}+\frac{\partial^{2} \bar{w}}{\partial \chi^{2}}\right)+\bar{q}(\chi, t)-m \ddot{w}=0
\end{gathered}
$$


where

$$
\begin{aligned}
h_{T}= & h_{1}+h_{2}+h_{3}, \quad \bar{w}=w / h_{T}, \quad \bar{\psi}=\psi / h_{T}, \quad \chi=x / L, \\
K_{11} & =\frac{h_{T}^{4}}{L^{4}}\left[E_{3}\left(\frac{h_{3}}{h_{T}}\right)^{2}-E_{1}\left(\frac{h_{1}}{h_{T}}\right)^{2}\right]^{2} /\left(4 E_{1} \frac{h_{1}}{h_{T}}+4 E_{3} \frac{h_{3}}{h_{T}}\right), \\
K_{12} & =\left[E_{3} \frac{h_{3}}{h_{T}} \frac{h_{c 2}^{2}}{h_{T}^{2}}\left(1+\frac{h_{3}}{2 h_{c 2}}\right)+E_{1} \frac{h_{1}}{h_{T}} \frac{h_{c 1}^{2}}{h_{T}^{2}}\left(1+\frac{h_{1}}{2 h_{c 1}}\right)\right]\left(\frac{h_{T}}{L}\right)^{4}, \\
K_{13} & =\left[E_{3} \frac{h_{3}}{h_{T}} \frac{h_{c 2}^{2}}{h_{T}^{2}}\left(1+\frac{h_{3}}{h_{c 2}}+\frac{h_{3}^{2}}{3 h_{c 2}^{2}}\right)+E_{1} \frac{h_{1}}{h_{T}} \frac{h_{c 1}^{2}}{h_{T}^{2}}\left(1+\frac{h_{1}}{h_{c 1}}+\frac{h_{1}^{2}}{3 h_{c 1}^{2}}\right)\right]\left(\frac{h_{T}}{L}\right)^{4}, \\
K_{15} & =\left[E_{1} \frac{h_{1}}{2 h_{T}}+E_{3} \frac{h_{3}}{2 h_{T}}\right]\left(\frac{h_{T}}{L}\right)^{4}, \\
K_{16} & =\left[E_{1} \frac{h_{1}^{2}}{h_{T}^{2}}-E_{3} \frac{h_{3}^{2}}{2 h_{T}^{2}}\right]\left(\frac{h_{T}}{L}\right)^{4}, \\
m & =\left(\rho_{1} h_{1}+\rho_{2} h_{2}+\rho_{3} h_{3}\right) h_{T}, \quad h_{2}=\left(h_{T} / L\right)^{2}, \quad q(\chi L, t)=\bar{q}(\chi, t) .
\end{aligned}
$$

Before closing this section, some remarks should be made on the possibility of making further simplifications by taking the face sheets to be very thin. The condition for locating the co-ordinate axis is equation (29). It, together with the relation $h_{2}=h_{c 1}+h_{c 2}$, leads to

$$
\begin{aligned}
& h_{c 1}=\left[E_{3} h_{3} /\left(E_{1} h_{1}+E_{3} h_{3}\right)\right] h_{2}, \\
& h_{c 2}=\left[E_{1} h_{1} /\left(E_{1} h_{1}+E_{3} h_{3}\right)\right] h_{2} .
\end{aligned}
$$

By thin face sheets is meant

$$
h_{1} / h_{c 1} \ll 1, \quad h_{3} / h_{c 2} \ll 1 .
$$

Upon calculating these ratios by using equations (37) and (38), it is seen that it is difficult to meet both the inequalities in equation (19) if $E_{1}$ and $E_{3}$ differ by even a factor of two or three. Hence, no thin face sheet assumptions are used here.

\section{FREQUENCY RESPONSE}

In this section, attention will be directed towards obtaining information on the response of the sandwich beam to a force consisting of a constant and a harmonic term. Of necessity, approximate means will be used. The spatial dependence of the integro-partial differential equations (35) and (36) will be eliminated by employing Galerkin's method. Then, the time aspect of the problem will be handled by means of the method of harmonic balance.

The transverse load, $\bar{q}$, is now assumed to be of the form

$$
\bar{q}(\chi, t)=a(\chi)\left(F_{0}+F_{1} \sin \Omega t\right),
$$

where

$$
a(\chi)=\left\{\begin{aligned}
0, & -1 / 2<\chi<-1 / 40 \\
20, & -1 / 40<\chi<1 / 40 \\
0, & 1 / 40<\chi<1 / 2,
\end{aligned}\right.
$$


$F_{0}, F_{1}$ are constants, and $\Omega$ denotes the driving frequency. The form of $a$ given by equation (41) is meant to simulate the spatial dependence of the electromagnetic force on the beam. On the assumption that steady-state conditions exist, the response is taken as

$$
\begin{gathered}
\bar{w}(\chi, t)=W(\chi) T_{1}(t), \\
\bar{\psi}(\chi, t)=\Psi(\chi) T_{2}(t) .
\end{gathered}
$$

In the Kovac, Anderson and Scott work [44], as well as in the experimental work of the present authors, the beams were clamped at both ends. Also, they were forced at their center, so that $\bar{w}$ and $\bar{\psi}$ are even and odd functions of $\chi$, respectively. With these items borne in mind, the following Galerkin functions, which are approximations to the first linear mode shape, are used:

$$
\begin{gathered}
W(\chi)=16\left(\chi^{2}-1 / 4\right)^{2}, \\
\Psi(\chi)=12 \sqrt{3} \chi\left(\chi^{2}-1 / 4\right)
\end{gathered}
$$

It should be noted that with this choice, the boundary term mentioned in connection with equation (7) is zero.

Upon using equations (40) through (45), Galerkin's method applied to equations (35) and (36) yields

$$
\begin{aligned}
& \left(\bar{K}_{11}+\overline{\bar{K}}_{12}-\bar{K}_{13}\right) T_{1}(t)+\bar{K}_{12} T_{2}(t)-H_{2} \int_{-\infty}^{t} Y_{2}(t-\tau) \mathrm{d} T_{2}(\tau)-\bar{H}_{2} \int_{-\infty}^{t} Y_{2}(t-\tau) \mathrm{d} T_{1}(\tau)=0 \\
& \left(\bar{K}_{15}-M\right) T_{1}(t)+H_{3} \int_{-\infty}^{t} Y_{2}(t-\tau) \mathrm{d} T_{2}(\tau)+H_{3} \int_{-\infty}^{t} Y_{2}(t-\tau) \mathrm{d} T_{1}(\tau)+F_{0}+F_{1} \sin \Omega t=0,(47)
\end{aligned}
$$

where

$$
\begin{array}{cl}
\bar{K}_{11}=-64 \cdot 5 K_{11}, & \bar{K}_{12}=-21 \cdot 7 K_{12}, \\
\bar{K}_{12}=-66 \cdot 5 K_{12}, & \bar{K}_{13}=-66 \cdot 5 K_{13}, \\
H_{2}=0 \cdot 514 h_{2}, & \bar{H}_{2}=1 \cdot 58 h_{2}, \\
\bar{K}_{15}=-23 \cdot 8 K_{15}, & H_{3}=1 \cdot 58 h_{2}, \\
\bar{H}_{3}=-4 \cdot 88 h_{2}, & M=0 \cdot 406 \mathrm{~m}, \\
F_{0}=F_{0} \int_{-1 / 2}^{1 / 2} a(\chi) W(\chi) \mathrm{d} \chi, & F_{1}=F_{1} \int_{-1 / 2}^{1 / 2} a(\chi) W(\chi) \mathrm{d} \chi
\end{array}
$$

The method of harmonic balance will now be employed to obtain frequency response information from equations (46) and (47). With generality in mind, the following timedependence of the response is assumed:

$$
\begin{gathered}
T_{1}(t)=W_{0}+W_{1} \sin \Omega t+W_{2} \cos \Omega t+W_{3} \sin 2 \Omega t+ \\
+W_{4} \cos 2 \Omega t+W_{5} \sin 3 \Omega t+W_{6} \cos 3 \Omega t \\
T_{2}(t)=V_{0}+V_{1} \sin \Omega t+V_{2} \cos \Omega t+V_{2} \cos \Omega t+V_{3} \sin 2 \Omega t \\
+V_{4} \cos 2 \Omega t+V_{5} \sin 3 \Omega t+V_{6} \cos 3 \Omega t
\end{gathered}
$$


Substituting equations (48) and (49) into equations (46) and (47), gives, on using the method of harmonic balance, with harmonics of the third order being retained,

$$
\begin{aligned}
& \left(\bar{K}_{11}+\bar{K}_{12}-\bar{K}_{13}\right)\left[W_{0}+W_{1} \sin \Omega t+W_{2} \cos \Omega t+W_{3} \sin 2 \Omega t+W_{4} \cos 2 \Omega t+\right. \\
& \left.+W_{5} \sin 3 \Omega t+W_{6} \cos 3 \Omega t\right]+\bar{K}_{12}\left[V_{0}+V_{1} \sin \Omega t+V_{2} \cos \Omega t+V_{3} \sin 2 \Omega t+\right. \\
& \left.+V_{4} \cos 2 \Omega t+V_{5} \sin 3 \Omega t+V_{6} \cos 3 \Omega t\right]-H_{2} \int_{-\infty}^{t} Y_{2}(t-\tau) \mathrm{d}\left(V_{0}+V_{1} \sin \Omega \tau+\right. \\
& \left.+V_{2} \cos \Omega \tau+V_{3} \sin 2 \Omega \tau+V_{4} \cos 2 \Omega \tau+V_{5} \sin 3 \Omega \tau+V_{6} \cos 3 \Omega \tau\right)- \\
& -\bar{H}_{2} \int_{-\infty}^{t} Y_{2}(t-\tau) \mathrm{d}\left(W_{0}+W_{1} \sin \Omega \tau+W_{2} \cos \Omega \tau+W_{3} \sin 2 \Omega \tau+W_{4} \cos 2 \Omega \tau+\right. \\
& \left.+W_{5} \sin 3 \Omega \tau+W_{6} \cos 3 \Omega \tau\right)=0
\end{aligned}
$$

and

$$
\begin{aligned}
& \bar{K}_{15}\left\{B_{0}+B_{1} \sin (\Omega t)+B_{2} \cos (\Omega t)+B_{3} \sin (2 \Omega t)+B_{4} \cos (2 \Omega t)+B_{5} \sin (3 \Omega t)+\right. \\
& +B_{6} \cos (3 \Omega t)+H_{3} \int_{-\infty}^{t} Y_{2}(t-\tau) \mathrm{d}\left\{V_{0}+V_{1} \sin (\Omega \tau)+V_{2} \cos (\Omega \tau)+V_{3} \sin (2 \Omega \tau)+\right. \\
& \left.+V_{4} \cos (2 \Omega \tau)+V_{5} \sin (3 \Omega \tau)+V_{6} \cos (3 \Omega \tau)\right\}+H_{3} \int_{-\infty}^{t} Y_{2}(t-\tau) \mathrm{d}\left\{W_{0}+W_{1} \sin (\Omega \tau)+\right. \\
& \left.+W_{2} \cos (\Omega \tau)+W_{3} \sin (2 \Omega \tau)+W_{4} \cos (2 \Omega \tau)+W_{5} \sin (3 \Omega \tau)+W_{6} \cos (3 \Omega \tau)\right\}+ \\
& +M \Omega^{2}\left(W_{1} \sin (\Omega t)+W_{2} \cos (\Omega t)+4 W_{3} \sin (2 \Omega t)+4 W_{4} \cos (2 \Omega t)+\right. \\
& \left.+9 W_{5} \sin (3 \Omega t)+9 W_{6} \cos (3 \Omega t)\right\}+F_{0}+F_{1} \sin (\Omega t)=0,
\end{aligned}
$$

where the $B_{i}$ 's are polynomials in the $W_{i}$ 's resulting from the non-linear term in the differential equation and are defined in the Appendix.

The integrals in equations (50) and (51) are treated by realizing that for a harmonic shearing strain, $\gamma(t)=\gamma_{0} \mathrm{e}^{j \Omega t}$, the shear stress, $S(t)$, can be represented by the complex modulus representation, resulting in

$$
S(t)=\left[G_{2}^{\prime}(\Omega)+\mathrm{j} G_{2}^{\prime \prime}(\Omega)\right] \gamma(t)=\int_{-\infty}^{t} Y_{2}(t-\tau) \mathrm{d} \gamma(\tau) .
$$

From this relation it can be shown that the relaxation function $Y_{2}$ is related to the complex modulus through the Fourier integrals

$$
\begin{aligned}
& G_{2}^{\prime}(\Omega)=\Omega \int_{-\infty}^{t} Y_{2}(\xi) \sin \Omega \xi \mathrm{d} \xi, \\
& G_{2}^{\prime \prime}(\Omega)=\Omega \int_{-\infty}^{t} Y_{2}(\xi) \cos \Omega \xi \mathrm{d} \xi .
\end{aligned}
$$

By means of equations (52) and (53), the various integrals in equations (50) and (51) can be evaluated. The results, on defining $\bar{K}_{T}=\bar{K}_{11}+\bar{K}_{12}-\bar{K}_{13}$, are 14 coupled, non-linear algebraic equations:

$$
\begin{gathered}
\bar{K}_{T} W_{0}+\bar{K}_{12} V_{0}-H_{2} G_{2}^{\prime}(0) V_{0}-\bar{H}_{2} G_{2}^{\prime}(0) W_{0}=0 \\
\bar{K}_{T} W_{1}+\bar{K}_{12} V_{1}-H_{2} G_{2}^{\prime}(\Omega) V_{1}-H_{2} G_{2}^{\prime \prime}(\Omega) V_{2}-\bar{H}_{2} G_{2}^{\prime}(\Omega) W_{1}+\bar{H}_{2} G_{2}^{\prime \prime}(\Omega) W_{2}=0
\end{gathered}
$$




$$
\begin{aligned}
& \bar{K}_{T} W_{2}+\bar{K}_{12} V_{2}-H_{2} G_{2}^{\prime \prime}(\Omega) V_{1}-H_{2} G_{2}^{\prime}(\Omega) V_{2}-\bar{H}_{2} G_{2}^{\prime \prime}(\Omega) W_{1}-\bar{H}_{2} G_{2}^{\prime}(\Omega) W_{2}=0, \\
& \bar{K}_{T} W_{3}+\bar{K}_{12} V_{3}-H_{2} G_{2}^{\prime}(2 \Omega) V_{3}+H_{2} G_{2}^{\prime \prime}(2 \Omega) V_{4} \\
& -H_{2} G_{2}^{\prime}(2 \Omega) W_{3}+\vec{H}_{2} G_{2}^{\prime \prime}(2 \Omega) W_{4}=0, \\
& \bar{K}_{T} W_{4}+\bar{K}_{12} V_{4}-H_{2} G_{2}^{\prime \prime}(\Omega) V_{3}-H_{2} G_{2}^{\prime}(2 \Omega) V_{4} \\
& -\bar{H}_{2} G_{2}^{\prime \prime}(2 \Omega) W_{3}-\bar{H}_{2} G_{2}^{\prime}(2 \Omega) W_{4}=0 \text {, } \\
& \bar{K}_{T} W_{5}+\bar{K}_{12} V_{5}-H_{2} G_{2}^{\prime}(3 \Omega) V_{5}+H_{2} G_{2}^{\prime \prime}(3 \Omega) V_{6}- \\
& -\bar{H}_{2} G_{2}^{\prime}(3 \Omega) W_{5}+\bar{H}_{2} G_{2}^{\prime \prime}(3 \Omega) W_{6}=0 \text {, } \\
& \bar{K}_{T} W_{6}+\bar{K}_{12} V_{6}-H_{2} G_{2}^{\prime \prime}(3 \Omega) V_{5}-H_{2} G_{2}^{\prime}(3 \Omega) V_{6}- \\
& -\bar{H}_{2} G_{2}^{\prime \prime}(3 \Omega) W_{5}-\bar{H}_{2} G_{2}^{\prime}(3 \Omega) W_{6}=0 \text {, } \\
& \bar{K}_{15} B_{0}+H_{3} G_{2}^{\prime}(0) V_{0}+\bar{H}_{3} G_{2}^{\prime}(0) W_{0}+F_{0}=0 \text {, } \\
& \bar{K}_{15} B_{1}+H_{3} G_{2}^{\prime}(\Omega) V_{1}-H_{3} G_{2}^{\prime \prime}(\Omega) V_{2}+\bar{H}_{3} G_{2}^{\prime}(\Omega) W_{1}- \\
& -\bar{H}_{3} G_{2}^{\prime \prime}(\Omega) W_{2}+M \Omega^{2} W_{1}+F_{1}=0 \text {, } \\
& \bar{K}_{15} B_{2}+H_{3} G_{2}^{\prime \prime}(\Omega) V_{1}+H_{3} G_{2}^{\prime}(\Omega) V_{2}+\bar{H}_{3} G_{2}^{\prime \prime}(\Omega) W_{1}+ \\
& +\bar{H}_{3} G_{2}^{\prime}(\Omega) W_{2}+M \Omega^{2} W_{2}=0, \\
& \bar{K}_{15} B_{3}+H_{3} G_{2}^{\prime}(2 \Omega) V_{3}-H_{3} G_{2}^{\prime \prime}(2 \Omega) V_{4}+\bar{H}_{3} G_{2}^{\prime}(2 \Omega) W_{3}- \\
& -\bar{H}_{3} G_{2}^{\prime \prime}(2 \Omega)+4 M \Omega^{2} W_{3}=0 \text {, } \\
& \bar{K}_{15} B_{4}+H_{3} G_{2}^{\prime \prime}(2 \Omega) V_{3}+H_{3} G_{2}^{\prime}(2 \Omega) V_{4}+\bar{H}_{3} G_{2}^{\prime \prime}(2 \Omega) W_{3}+ \\
& +\vec{H}_{3} G_{2}^{\prime}(2 \Omega) W_{4}+4 M \Omega^{2} W_{4}=0 \text {, } \\
& \bar{K}_{15} B_{5}+H_{3} G_{2}^{\prime}(3 \Omega) V_{5}+H_{3} G_{2}^{\prime \prime}(3 \Omega) V_{6}-\bar{H}_{3} G_{2}^{\prime}(3 \Omega) W_{5}- \\
& -\bar{H}_{3} G_{2}^{\prime \prime}(3 \Omega) W_{6}+9 M \Omega^{2} W_{5}=0, \\
& \bar{K}_{15} B_{6}+H_{3} G_{2}^{\prime \prime}(3 \Omega) V_{5}+H_{3} G_{2}^{\prime}(3 \Omega) V_{6}+\bar{H}_{3} G_{2}^{\prime \prime}(3 \Omega) W_{5}+ \\
& \bar{H}_{3} G_{2}^{\prime}(3 \Omega) W_{6}+9 M \Omega^{2} W_{6}=0 \text {. }
\end{aligned}
$$

\section{NUMERICAL RESULTS}

Though formidable in appearance, the algebraic equations (54) through (67) can be handled numerically. The method used here was one developed by Brown and Conte [50, 51]. It is a gradient method with the gradient computed numerically rather than having to enter the partial derivatives explicitly. The $W_{i}$ and $V_{i}$ are functions of frequency and frequencyresponse curves for r.m.s. transverse displacement at mid-span were calculated from equation (42). Results were obtained for the symmetric beams reported on by Kovac, Anderson and Scott [44], and for unsymmetric beams not previously investigated. Complex modulus data was supplied by the B. F. Goodrich Co., in connection with the work in reference [44].

Results show that superharmonic response is present, but it does not have a significant effect on the frequency response curve. Figure 4 shows results for the beam of Figure 2. Included in Figure 4 is the theoretical ratio of the magnitude of the $2 \Omega$ component of response to the magnitude of the $\Omega$ component as a function of forcing frequency. As indicated, the ratio is largest when forcing the beam near one-half its resonant frequency. Curves similar to those in Figure 4 were found for the unsymmetrical beams to be reported on later. Results 


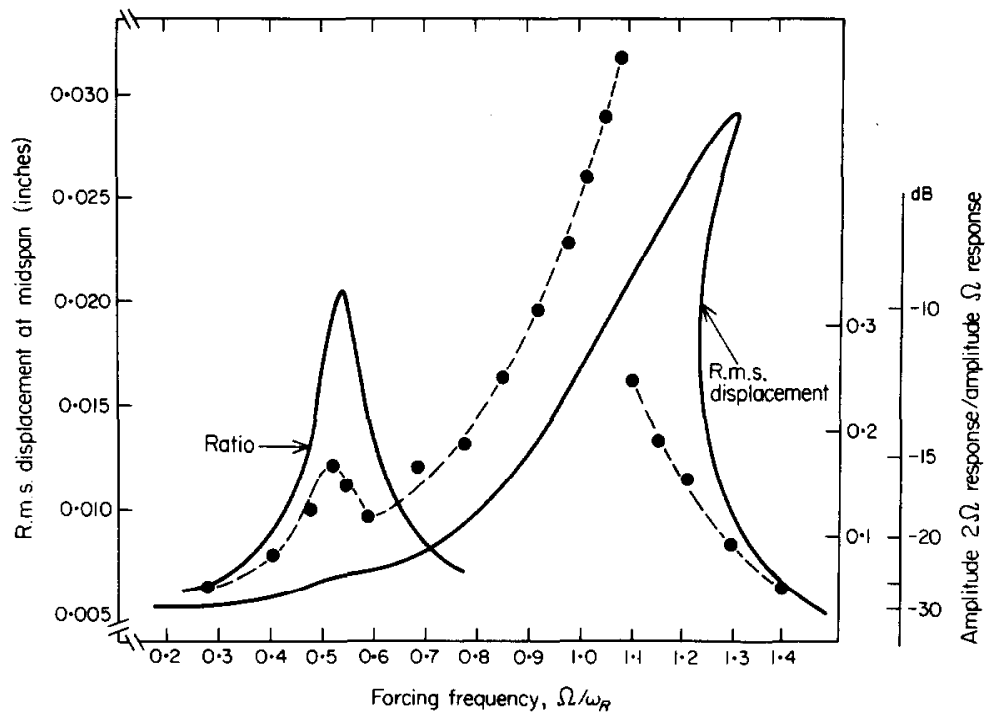

Figure 4. Superharmonic response of symmetric three-layer beam. _- Theory; -.- --, experiment (reference [44]); $\omega_{R}=$ linear resonant frequency.

show that for both symmetric and unsymmetric beams, the superharmonic response is much more pronounced if the bias force is larger than the harmonic force. However, it seems clear that some other effect was responsible for the large increase in response amplitude originally observed when forcing the beam at one-half its resonant frequency. This will be discussed at some length in the paper reporting the experimental results.

\section{ACKNOWLEDGMENT}

The authors would like to thank the referees for their thorough review of the manuscript and several helpful suggestions.

\section{REFERENCES}

1. L. M. HaBIP 1965 Applied Mechanics Reviews 18, 93-98. A survey of modern developments in the analysis of sandwich structures.

2. Y.-Y. Yu 1959 Journal of Applied Mechanics 81, 415-421. A new theory of elastic sandwich plates, one dimensional case.

3. Y. -Y. Yu 1960 Journal of Aerospace Science 27, 272-282. Flexural vibrations of elastic sandwich plates.

4. Y. -Y. Yu 1960 Journal of Aerospace Science 27, 894-900. Simplified vibration analysis of elastic sandwich plates.

5. D. KRAJCINOVIC 1972 Journal of Applied Mechanics 39, 773-778. Sandwich beam analysis.

6. D. KRAJCINOVIC 1973 American Institute of Aeronautics and Astronautics 10, 1265-1266. Vibrations of laminated beams.

7. G. M. Folie 1972 Revue Roum. Des Sciences Techniques Série De Mechanique Appliquee, 17, 19-36. Transverse bending of sandwich plates.

8. M. -C. ChAN and Y. K. CheUNG 1972 International Journal of Mechanical Science 14, 399-406. Static and dynamic analysis of multi-layered sandwich plates.

9. K. M. AHMED 1971 Journal of Sound and Vibration 18, 61-74. Free vibration of curved sandwich beams by the method of finite elements.

10. A. V. KRishna MURTY and R. P. Shimpi 1974 Journal of Sound and Vibration 36, 273-284. Vibrations of laminated beams. 
11. M. BeN-Amoz 1975 International Journal of Engineering Science 13, 43-55. On wave propagation in laminated composites I. Propagation parallel to the laminates.

12. R. Plunkett 1974 American Society of Mechanical Engineers Paper 74-DE-10. Design for vibration control by damping.

13. R. A. DiT ARANTo 1965 Journal of Applied Mechanics 32, 881-886. Theory of vibratory bending for elastic and viscoelastic layered finite-length beams.

14. R. A. DiTaranto and W. Blasingame 1967 Journal of Engineering for Industry 89, 633-638. Composite damping of vibrating sandwich beams.

15. I. W. JONES, V. C. SALERNO and A. SAVACCHIO 1967 Journal of Engineering for Industry 89, 438444. An analytical and experimental evaluation of the damping capacity of sandwich beams with viscoelastic cores.

16. T. P. YIN, T. J. KeLLY and J. E. BARRY 1967 Journal of Engineering for Industry 89, 773-784. A quantitative evaluation of constrained-layer damping.

17. D. J. Mfan and S. MAR Kus 1969. Journal of Sound and Vibration 10, 163-175. The forced vibration of a three-layer damped sandwich beam with arbitrary boundary conditions.

18. D. J. MEAD and S. MARKus 1970 Journal of Sound and Vibration 12, 99-112. Loss factors and resonant frequencies of encastré damped sandwich beams.

19. D. J. Mead and R. A. DiTaranto 1972 Journal of Engineering for Industry 94, 175-180. Resonance criteria of a damped three-layer beam.

20. Y. P. Lu and B. E. Douglas 1974 Journal of Sound and Vibration 32, 513-516. On the forced vibrations of three-layer damped sandwich beams.

21. P. GROOTENHUIS 1970 Journal of Sound and Vibration 11, 421-433. The control of vibrations with viscoelastic materials.

22. J. A. Agbasieke and P. Grovi enhuis 1968 Journal of Mechanical Engineering Science 10, 269281. Flexural vibration of symmetrical multi-layer beams with viscoelastic damping.

23. B. C. NAKRA and P. GROOTENHUIS 1972 Journal of Engineering for Industry 94, 81-86. Structural damping using a four-layer sandwich.

24. P. GROOTENHUIS 1969 Environmental Engineering 38, 7-13. Vibration control with viscoelastic materials.

25. M. -J. YAN and E. H. Dowfir. 1972 Journal of Applied Mechanics 39, 1041-1046. Governing equations for vibrating constrained-layer damping sandwich plates and beams.

26. M. -J. YAN and E. H. DOWELl 1973 American Institute of Aeronautics and Astronautics Journal 11, 388-390. High-damping measurements and a preliminary evaluation of an equation for constrained layer damping.

27. N. T. ASNANı and B. C. NAKRA 1972 Journal of the Aeronautical Society of India 24, 288-294. Forced vibration analysis of sandwich beams with viscoelastic core.

28. H. BRAUNISCH 1970 Acustica 22, 136-144. Vibration damping by three-layered sandwich systems.

29. M. P. ChandraseKharan and A. Ghosh 1974 Journal of Sound and Vibration 37, 1-15. Damping characteristics of elastic-viscoelastic composite shafts.

30. R. A. DiT ARANTO and J. R. MCGRAW, JR. 1969 Journal of Engineering for Industry 91, 1081-1090. Vibratory bending of damped laminated plates.

31. P. D. EMERSON 1974 Journal of Engineering for Industry 96, 299-303. Application of constrainedlayer damping to control noise in machine parts.

32. D. I. G. JoNES and M. L. PARIN 1972 Journal of Sound and Vibration 24, 201-210. Technique for measuring damping properties of thin viscoelastic layers.

33. O. Markus and S. Markus 1974 Acustica 31, 132-138. Damping properties of sandwich beam with local shearing prevention.

34. B. C. NAKRA 1973 Journal of the Aeronautical Society of India 25, 35-39. Vibration analysis of sandwich beam with constrained viscoelastic layers on both sides.

35. Y. V. K. Sadasiva RaO 1974 Journal of Sound and Vibration 32, 175-187. Vibrations of a dual core sandwich beam.

36. P. J. ToRviK and D. F. STRICKLANd 1972 Journal of the Acoustical Society of America 51A, 985991. Damping additions for plates using constrained viscoelastic layers.

37. C. -H. Ho, R. A. ScotT and J. G. EISLEY 1975 International Journal of Non-Linear Mechanics 10, 113-127. Nonplanar, non-linear oscillation of a beam-I. Forced motions.

38. E. REISSNER 1948 Journal of Aerospace Science 15, 435-440. Finite deflections of sandwich plates.

39. Y. -Y. YU 1962 Journal of Aerospace Science 29, 886-888. Comment on influence of transverse shear on non-linear vibrations of sandwich beams with honey-comb cores.

40. Y. -Y. YU 1962 Journal of the Acoustical Society of America 34, 1176-1183. Non-linear flexural vibrations of sandwich plates. 
41. G. A. WEMPNER and J. L. BAYLOR 1965 International Journal of Solids and Structures 1, 157-177. General theory of sandwich plates with dissimilar facings.

42. L. M. HABIP 1967 International Journal of Solids and Structures 3, 207-215. Moderately large deflections of asymmetrical layered elastic plates.

43. C. W. BERT 1973 Journal of Applied Mechanics 40, 452-458. Non-linear vibration of a rectangular plate arbitrarily laminated of anisotropic material.

44. E. J. KovaC, W. J. ANDERson and R. A. Scott 1971 Journal of Sound and Vibration 17, 25-39. Forced non-linear vibrations of a damped sandwich beam.

45. J. A. BeNNETT and J. G. EISLEY 1970 American Institute of Aeronautics and Astronautics Journal 8, 734-739. A multiple-degree-of-freedom approach to non-linear beam vibrations.

46. L. MeIrovitch 1975 Elements of Vibration Analysis. New York: McGraw-Hill Book Company, Inc. See p. 380.

47. Y. C. Fung 1965 Foundations of Solid Mechanics. Englewood Cliffs: Prentice-Hall. See p. 91.

48. M. W. HYER 1974 Ph.D. Dissertation, University of Michigan. Non-linear vibrations of threelayer beams.

49. H. -N. Chu and G. Herrmann 1956 Journal of Applied Mechanics 23, 532-540. Influence of large amplitudes on free flexural vibrations of rectangular elastic plates.

50. K. M. BRown and S. D. CONTE 1967 Proceedings of the 22nd National Conference of the ACM, 111-114. The Solution of Simultaneous Nonlinear Equations.

51. G. D. BYRNE and C. H. HALl (Editors) 1973 Numerical Solutions of Systems of Algebraic Equations. New York: Academic Press.

\section{APPENDIX}

$$
\begin{aligned}
B_{0}= & C_{0} W_{0}+(1 / 2)\left(C_{1} W_{1}+C_{2} W_{2}+C_{3} W_{3}+C_{4} W_{4}+C_{5} W_{5}+C_{6} W_{6}\right) \\
B_{1}= & C_{1} W_{0}+C_{0} W_{1}+(1 / 2)\left(-C_{4} W_{1}+C_{3} W_{2}+C_{2} W_{3}-\right. \\
& \left.-C_{6} W_{3}-C_{1} W_{4}+C_{5} W_{4}+C_{4} W_{5}-C_{8} W_{5}-C_{3} W_{6}+C_{7} W_{6}\right) \\
B_{2}= & C_{2} W_{0}+C_{0} W_{2}+(1 / 2)\left(C_{3} W_{1}+C_{4} W_{2}+C_{1} W_{3}+\right. \\
& \left.+C_{5} W_{3}+C_{2} W_{4}+C_{6} W_{4}+C_{3} W_{5}+C_{7} W_{5}+C_{4} W_{6}+C_{8} W_{6}\right) \\
B_{3}= & C_{3} W_{0}+C_{0} W_{3}+(1 / 2)\left(C_{2} W_{1}-C_{6} W_{1}+C_{1} W_{2}+\right. \\
& \left.+C_{5} W_{2}-C_{8} W_{3}+C_{7} W_{4}+C_{2} W_{5}-C_{10} W_{5}-C_{1} W_{6}+C_{9} W_{6}\right) \\
B_{4}= & C_{4} W_{0}+C_{0} W_{4}+(1 / 2)\left(-C_{1} W_{1}+C_{5} W_{1}+C_{2} W_{2}+\right. \\
& \left.+C_{6} W_{2}+C_{7} W_{3}+C_{8} W_{4}+C_{1} W_{5}+C_{9} W_{5}+C_{2} W_{6}+C_{10} W_{6}\right) \\
B_{5}= & C_{5} W_{0}+C_{0} W_{5}+(1 / 2)\left(C_{4} W_{1}-C_{8} W_{1}+C_{3} W_{2}+\right. \\
& \left.+C_{7} W_{2}+C_{2} W_{3}-C_{10} W_{3}+C_{1} W_{4}+C_{9} W_{4}+C_{11} W_{6}-C_{12} W_{5}\right) \\
B_{6}= & C_{6} W_{0}+C_{0} W_{6}+(1 / 2)\left(-C_{3} W_{1}+C_{7} W_{1}+C_{4} W_{2}+\right. \\
& \left.+C_{8} W_{2}-C_{1} W_{3}+C_{9} W_{3}+C_{2} W_{4}+C_{10} W_{4}+C_{11} W_{5}+C_{12} W_{6}\right), \\
C_{0}= & W_{0}^{2}+(1 / 2)\left(W_{1}^{2}+W_{2}^{2}+W_{3}^{2}+W_{4}^{2}+W_{5}^{2}+W_{6}^{2}\right) \\
C_{1}= & 2 W_{0} W_{1}-W_{1} W_{4}+W_{2} W_{3}-W_{3} W_{6}+W_{4} W_{5} \\
C_{2}= & 2 W_{0} W_{2}+W_{1} W_{3}+W_{2} W_{4}+W_{3} W_{5}+W_{4} W_{6} \\
C_{3}= & 2 W_{0} W_{3}+W_{1} W_{2}-W_{1} W_{6}+W_{2} W_{5}, \\
C_{4}= & (1 / 2)\left(W_{2}^{2}-W_{1}^{2}\right)+2 W_{0} W_{4}+W_{1} W_{5}+W_{2} W_{6} \\
C_{5}= & 2 W_{0} W_{5}+W_{1} W_{4}+W_{2} W_{3}, \\
&
\end{aligned}
$$




$$
\begin{aligned}
& C_{6}=2 W_{0} W_{6}-W_{1} W_{3}+W_{2} W_{4}, \\
& C_{7}=W_{1} W_{6}+W_{2} W_{5}+W_{3} W_{4}, \\
& C_{8}=(1 / 2)\left(W_{4}^{2}-W_{3}^{2}\right)+W_{2} W_{6}-W_{1} W_{5}, \\
& C_{9}=W_{3} W_{6}+W_{4} W_{5}, \\
& C_{10}=W_{4} W_{6}-W_{3} W_{5}, \\
& C_{11}=W_{5} W_{6}, \\
& C_{12}=\frac{1}{2}\left(W_{6}^{2}-W_{5}^{2}\right) .
\end{aligned}
$$

